\title{
Data quality problems undermine analyses of endotherm upper critical temperatures
}

\begin{abstract}
In an analysis of avian and mammalian thermal tolerances recently published in this journal, Khaliq et al. (2015) reported that endotherm thermal niches are phylogenetically conserved in tropical, but not temperate, regions. However, closer examination of the data upon which this analysis was based reveals that many of the upper critical temperature (UCT) data are not valid. Approximately $55 \%$ and $42 \%$ of avian and mammalian UCT data, respectively, originated from studies in which animals were not exposed to air temperatures high enough to elicit an increase in metabolic rate above minimum levels; the cited UCT values are merely the highest air temperatures at which measurements took place. An additional $18 \%$ and $25 \%$ of avian and mammalian UCT data, respectively, represent values based on just one individual per species and/or measurements at too few air temperatures above the thermoneutral zone (TNZ) to reliably estimate the UCT.
\end{abstract}

Keywords: birds; data inclusion criteria; mammals; thermal tolerance; thermoneutral zone; upper critical limit of thermoneutrality

Several recent studies examining global variation in the thermal tolerances of endotherms have focused on the upper and lower limits of the thermoneutral zone (TNZ), the range of air temperatures over which resting metabolic rate is minimal and increments associated with heat production or active heat dissipation are absent. One such study was recently published in this journal; Khaliq and colleagues partitioned variation in thermal traits of mammals and birds into phylogenetic and environmental (i.e., adaptive) components, and reported phylogenetic niche conservatism in tropical, but not temperate, regions (Khaliq et al., 2015).

Our purpose here is to highlight serious flaws in the data set upon which the conclusions of Khaliq et al. (2015) are based. The problems concern the upper critical temperature (UCT), the air temperature representing the upper boundary of the TNZ (also known as the upper critical limit of thermoneutrality). A second variable included in the analysis, TNZ breadth, was calculated as the difference between the UCT and the lower critical temperature, and so the shortcomings of the UCT data set apply equally to the TNZ breadth data.

The fundamental problem with the UCT and TNZ breadth data analysed by Khaliq et al. (2015) is that many of these data simply do not exist, as they originate from empirical studies in which metabolic rate was not measured at air temperatures high enough to elicit the increase that defines the UCT. Khaliq et al. (2015) included mammalian and avian UCT data as defined in an earlier study (Khaliq et al., 2014); the correct definition of UCT is provided in the Electronic Supplementary Material of the latter paper. However, for many UCT data included in their analyses this definition was not followed.

We examined the original papers cited as the sources of 237 of 255 avian data and 284 of 295 mammalian data provided in Appendix S1 of Khaliq et al. (2015). On the basis of visual inspection of the relationship between resting metabolic rate and air temperature, we allocated each datum to one of the following categories (Appendix S1, Supporting Information): 
1) Good data - increase in metabolic rate above the TNZ, with a clear inflection point defining the UCT based on data for at least two individuals per species (Figure 1A).

2) Insufficient data - some evidence of an increase in metabolic rate above the TNZ, but based on only a single individual and/or measurements at too few air temperatures to reliably estimate a UCT (Figure 1B). Although it is often possible to fit piecewise regression models to these data sets using software such as the $R$ package 'segmented' (Muggeo, 2009), inflection points identified in this way are highly sensitive to whether or not sufficient data exist at higher air temperatures to reliably estimate the slope (Figure 2).

3) No UCT - by Khaliq et al.'s (2014) definition the data do not exist; metabolic rate was not measured at air temperatures high enough to elicit an increase above thermoneutral levels (Figure 1C).

Of the 284 mammalian UCT data we checked, good data (Category 1) accounted for only $33.1 \%$ of the original data set (94 species). A further $25.4 \%$ (72 species) had insufficient data (Category 2), and for the remaining $41.5 \%$ (118 species) the UCT did not exist (Category 3).

Of the 237 avian UCT data we checked, good data (Category 1) accounted for only $27.0 \%$ of the original data set (64 species). A further $18.1 \%$ (43 species) had insufficient data (Category 2), and for the remaining $54.9 \%$ (130 species) the UCT did not exist (Category 3). Approximately one quarter of the avian UCT data are valid, and over half effectively do not exist.

The reasons why the correct UCT definition was not applied by Khaliq et al. when compiling the data set are not clear. In at least one instance, unambiguous statements by the authors of an original study that no UCT was reached were apparently overlooked. In their study of the thermal physiology of estrildid finches, Marschall \& Prinzinger (1991) clearly indicated that three species (Estrilda melpoda, Padda oryzivora, and Taeniopygia guttata) did not reach a UCT. Nevertheless, Khaliq et al. (2015) included UCT values for these three species in their data set. In other instances, original papers admittedly do contain potentially ambiguous phrases such as "Thermoneutrality extended from XX to YY ${ }^{\circ} \mathrm{C}$ " (McNab, 2000, 2001; McNab, 2003), where $\mathrm{YY}^{\circ} \mathrm{C}$ was simply the highest air temperature at which metabolic rate was measured. However, even cursory examination of the figures in these papers reveals that no UCT was reached (see Figure 1C for an example of such a data set). 


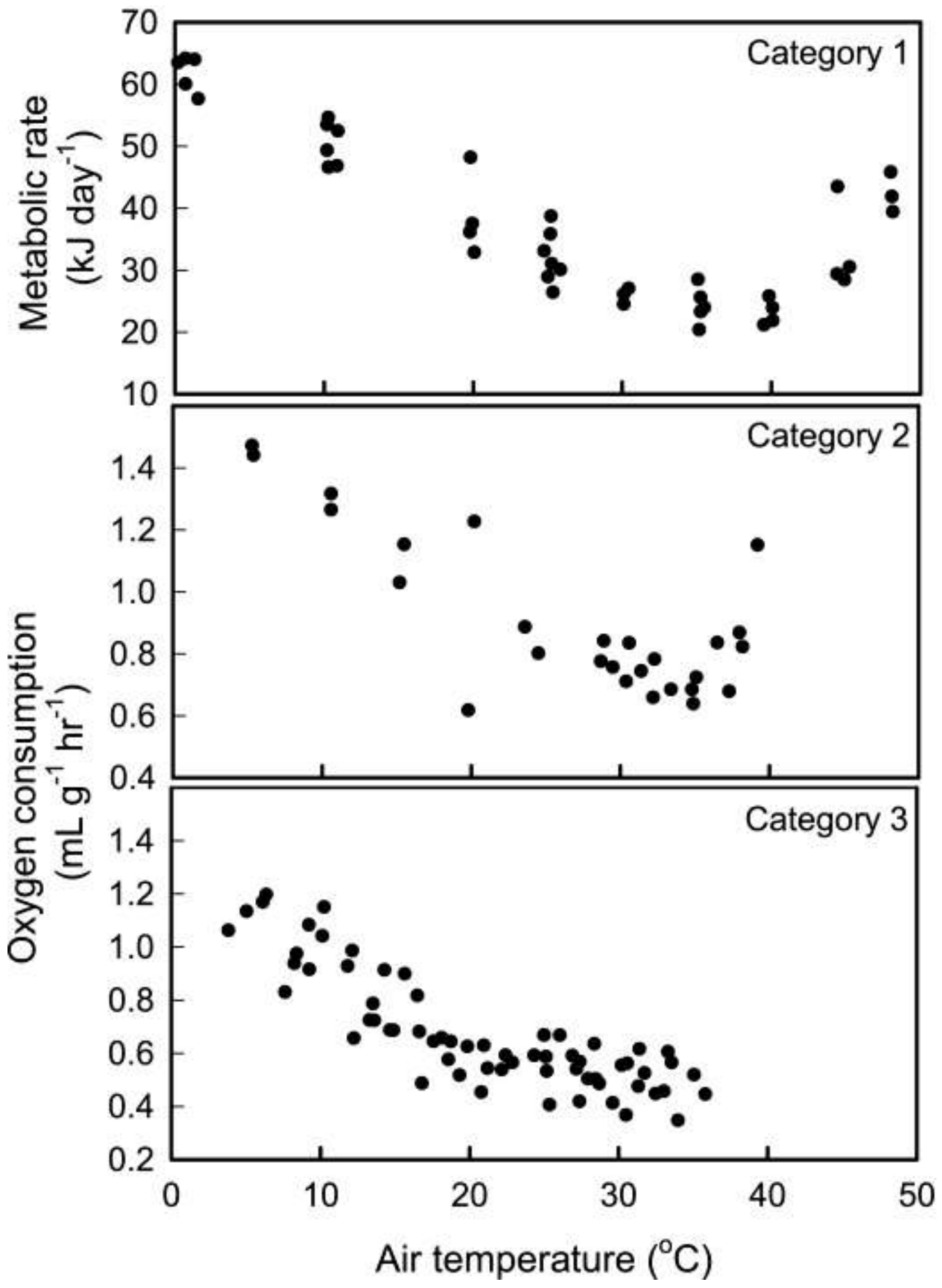

Figure 1. Examples of data included by Khaliq et al. (2015) illustrating our three categories of upper critical temperature (UCT) data quality. Category 1 (top panel): metabolic rate in Dunn's larks (Eremalauda dunni; data from Tieleman et al., 2002). In this case, metabolic rate is measured at two air temperatures obviously above the thermoneutral zone (TNZ), allowing for accurate estimation of a UCT. Category 2 (middle panel): night-time oxygen consumption in grey-necked wood-rails (Aramides cajanea; data from McNab \& Ellis, 2006). The increase in metabolic rate above the TNZ involves only a single data point and the UCT can hence not be estimated accurately. Category 3 (bottom panel): night-time oxygen consumption in Nicobar pigeons (Caloenas nicobarica; data from McNab, 2000). There is no increase in oxygen consumption associated with a UCT; the value of $37{ }^{\circ} \mathrm{C}$ included in the data set analysed by Khaliq et al. (2015) is simply the highest air temperature at which oxygen consumption was measured. Data for E. dunni, A. cajanea and C. nicobarica were extracted from original figures using DigitizeIt software (http://www.digitizeit.de) solely for plotting them here. 


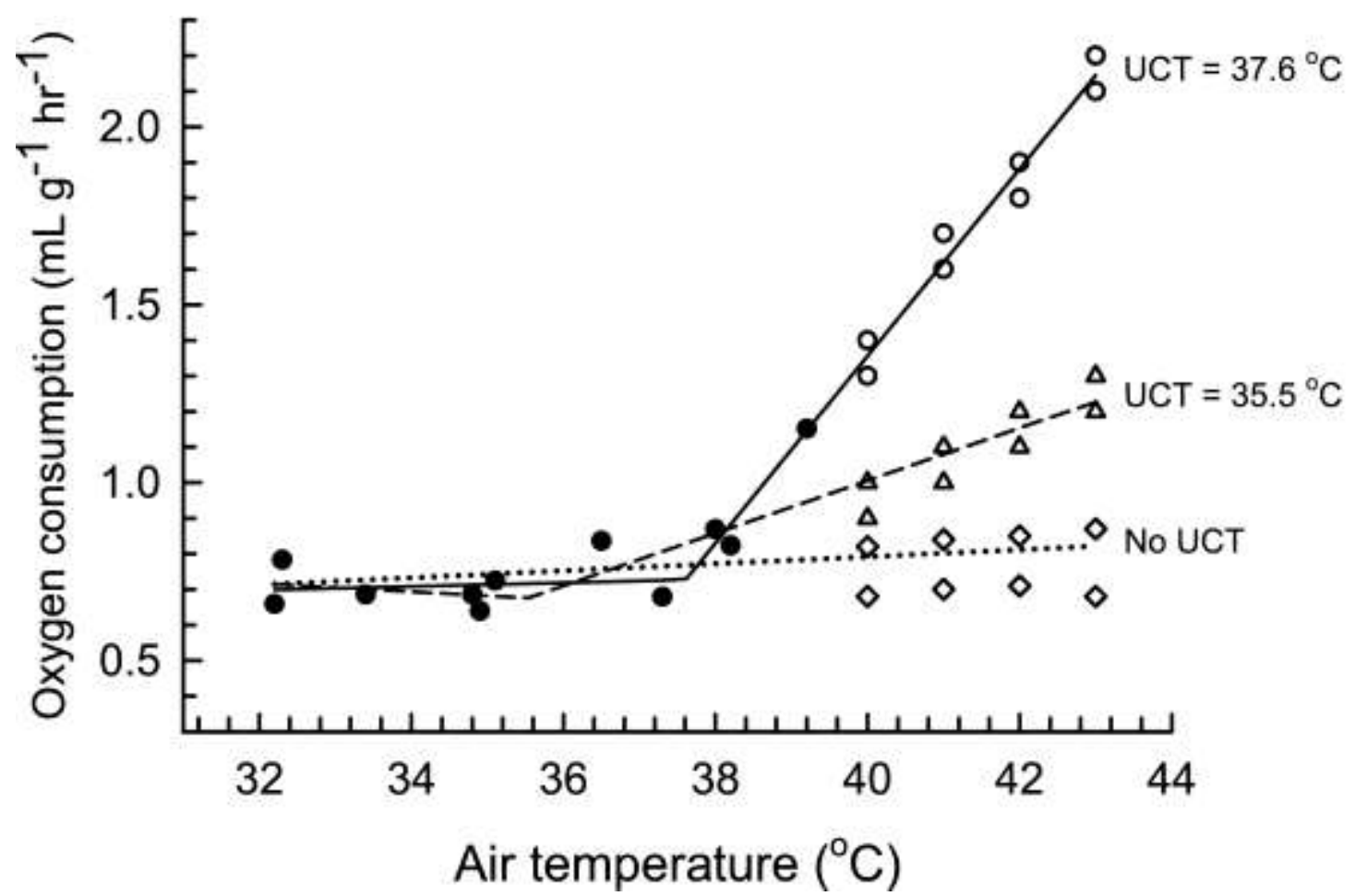

Figure 2. Resting metabolic rate data measured at air temperatures $\left(T_{\mathrm{a}}\right)$ well above the thermoneutral zone are necessary for reliable estimates of the upper critical temperature (UCT). McNab \& Ellis (2006) measured oxygen consumption in grey-necked wood-rails (Aramides cajanea) over a $\mathrm{T}_{\mathrm{a}}$ range extending up to $c .39^{\circ} \mathrm{C}$ (actual data shown as filled circles); these are a subset of the data shown in Fig. 1 (middle panel). The open symbols are hypothetical data that we have added in order to illustrate why data at a range of $T_{\mathrm{a}}$ above the thermoneutral zone are essential for accurate estimation of the UCT. The three lines are regression models fitted to the data sets (actual data and hypothetical data combined) using the $R$ package 'segmented'. The hypothetical data shown by open circles fall along the line suggested by the single data point that $\mathrm{McNab}$ and Ellis collected at $T_{\mathrm{a}}=39{ }^{\circ} \mathrm{C}$; in this instance, the estimated UCT is at $T_{\mathrm{a}}=37.6{ }^{\circ} \mathrm{C}$ (solid line), close to the value used by Khaliq et al. The hypothetical data shown by open triangles, in contrast, illustrate a situation where the actual slope is shallower than suggested by the single datum at $T_{\mathrm{a}}=39{ }^{\circ} \mathrm{C}$; in this instance, the UCT is nearly $2{ }^{\circ} \mathrm{C}$ lower, at $T_{\mathrm{a}}=35.5^{\circ} \mathrm{C}$ (dashed line). The third set of hypothetical data, shown by open diamonds, illustrates a situation characteristic of taxa such as caprimulgid birds (e.g. Dawson \& Fisher, 1969; O'Connor et al., in press) where RMR remains low even at very high $T_{\mathrm{a}}$; in such instances, no UCT occurs (dotted line).

Overall, just $\sim 30 \%$ of the mammalian and avian UCT data that formed the basis for the analyses by Khaliq et al. (2015) can be considered reliable estimates of the upper boundary of the TNZ. In our opinion, these data quality problems fatally undermine these authors' qualitative and quantitative conclusions about the effects of phylogeny and environment on UCT and TNZ breadth. We recommend the authors repeat these analyses using a more constrained data set to which appropriate data inclusion criteria are rigorously applied.

Andrew E. McKechnie ${ }^{1, *}$

Brittney H. Coe ${ }^{2}$

Alexander R. Gerson ${ }^{3}$

Blair O. Wolf ${ }^{2}$

${ }^{1}$ DST-NRF Centre of Excellence at the Percy FitzPatrick Institute, Department of Zoology and Entomology, University of Pretoria, Private Bag X20, Hatfield 0028, South Africa 
${ }^{2}$ UNM Biology Department, University of New Mexico, MSC03-2020, Albuquerque, NM 87131-0001, USA

${ }^{3}$ Department of Biology, University of Massachusetts, Amherst, MA 01003, USA

*Address for correspondence

aemckechnie@zoology.up.ac.za

\section{References}

Dawson, W.R. \& Fisher, C.D. (1969) Responses to temperature by the spotted nightjar (Eurostopodus guttatus). Condor, 71, 49-53.

Khaliq, I., Hof, C., Prinzinger, R., Böhning-Gaese, K. \& Pfenninger, M. (2014) Global variation in thermal tolerances and vulnerability of endotherms to climate change. Proceedings of the Royal Society of London B: Biological Sciences, 281, 20141097.

Khaliq, I., Fritz, S.A., Prinzinger, R., Pfenninger, M., Böhning - Gaese, K. \& Hof, C. (2015) Global variation in thermal physiology of birds and mammals: evidence for phylogenetic niche conservatism only in the tropics. Journal of Biogeography, 42, 2187-2196.

Marschall, U. \& Prinzinger, R. (1991) Vergleichende ökophysiologie von fünf prachtfinkenarten (Estrildidae). Journal für Ornithologie, 132, 319-323.

McNab, B.K. (2000) The influence of body mass, climate, and distribution on the energetics of South Pacific pigeons. Comparative Biochemistry and Physiology A, 127, 309-329.

McNab, B.K. (2001) Energetics of toucans, barbets and a hornbill: implications for avian frugivory. Auk, 118, 916-933.

McNab, B.K. (2003) The energetics of New Zealand's ducks. Comparative Biochemistry and Physiology Part A: Molecular \& Integrative Physiology, 135, 229-247.

McNab, B.K. \& Ellis, H.I. (2006) Flightless rails endemic to islands have lower energy expenditures and clutch sizes than flighted rails on islands and continents. Comparative Biochemistry and Physiology Part A: Molecular \& Integrative Physiology, 145, 295-311.

Muggeo, V.M.R. (2009) Segmented: an R package to fit regression models with broken-line relationships.

O'Connor, R.S., Wolf, B.O., Brigham, R.M. \& McKechnie, A.E. (in press) Avian thermoregulation in the heat: efficient evaporative cooling in two southern African nightjars. Journal of Comparative Physiology $B$,

Tieleman, B.I., Williams, J.B. \& Buschur, M.E. (2002) Physiological adjustments to arid and mesic environments in larks (Alaudidae). Physiological and Biochemical Zoology, 75, 305-313. 
Appendix S1. Body mass and upper critical temperature (UCT) data used by Khaliq et al. (2015), with each datum categorised as 1) good, 2) insufficient data ("Ins. data") or 3) no UCT, using criteria provided in the text. Data with "-----" listed under Category are those for which we were unable to check the original data set, typically because either we were unable to obtain the original source, or because an incorrect reference was provided by Khaliq et al. (2015). References are those provided in the Supporting Information of Khaliq et al. (2015).

\begin{tabular}{lcclc}
\hline Species & $\begin{array}{c}\text { Body Mass } \\
(\mathbf{g})\end{array}$ & UCT $\left({ }^{\circ} \mathbf{C}\right)$ & References & Category \\
\hline Birds & & & & \\
Buteo jamaicensis & 1475 & 34 & Wasser, 1986, Hayes \& Gessman, 1980 & No UCT \\
Aquila chrysaetos & 4320 & 35 & Hayes \& Gessman, 1980 & No UCT \\
Buteo buteo & 1012 & 35 & Herpel, 2007 & ---- \\
Anas castanea & 483.3 & 37 & McNab, 2003 & NCT \\
Anas rhynchotis & 508 & 37 & McNab, 2003 UCT \\
Aythya novaeseelandiae & 488.4 & 33 & McNab, 2003 & No UCT \\
Tadorna variegata & 1193.6 & 33 & McNab, 2003 & Good \\
Anas aucklandica & 373.1 & 37 & McNab, 2003 & No UCT \\
Anas gracilis & 393.7 & 39 & McNab, 2003 & No UCT \\
Hymenolaimus malacorhynchos & 717.1 & 38 & McNab, 2003 & No UCT \\
Anas penelope & 723 & 22 & Jilg, 2006 & ----- \\
Anas rubripes & 1263 & 22 & Wooley et al., 1977 & No UCT \\
Somateria mollissima & 1660 & 25 & Richman \& Lovvorn, 2011, Hawkins et al., 2000 & No UCT \\
Clangula hyemalis & 490 & 26 & Jenssen \& Ekker, 1989 & ------ \\
Collocalia vanikorensis & 11.6 & 34 & McNab \& Bonaccorso, 1995 & Good \\
Collocalia esculenta & 6.8 & 34 & McNab \& Bonaccorso, 1995 & Good
\end{tabular}




$\begin{array}{lccl}\text { Aegotheles cristatus } & 45.56 & 35 & \text { Doucette \& Geiser, 2008 } \\ \text { Eurostopodus argus } & 88 & 47 & \text { Dawson \& Fisher, 1969 } \\ \text { Caprimulgus macrurus } & 68.6 & 36 & \text { McNab \& Bonaccorso, 1995 } \\ \text { Eurostopodus mystacalis } & 162 & 37 & \text { McNab \& Bonaccorso, 1995 } \\ \text { Phalaenoptilus nuttallii } & 40 & 44 & \text { Bartholomew } \text { et al., 1962 } \\ \text { Caprimulgus vociferus } & 46 & 37 & \text { Lane } \text { et al., 2004 } \\ \text { Chordeiles minor } & 72 & 39 & \text { Lasiewski } \text { et al., 1970, Lasiewski \& Dawson, } 1964 \\ \text { Podargus strigoides } & 380.3 & 38 & \text { McNab \& Bonaccorso, 1995 } \\ \text { Cepphus grylle } & 342.2 & 22 & \text { Gabrielsen } \text { et al., 1988 } \\ \text { Alle alle } & 152.5 & 20 & \text { Gabrielsen } \text { et al., 1991 } \\ \text { Uria lomvia } & 819 & 18 & \text { Bryant \& Furness, 1995, Gabrielson } \text { et al., } 1987 \\ \text { Pluvialis apricaria } & 151 & 30 & \text { Daan } \text { et al., 1990 } \\ \text { Haematopus } \text { ostralegus } & 385.4 & 30 & \text { Heyer, 1995 } \\ \text { Rissa tridactyla } & 365 & 18 & \text { Gabrielson } \text { et al., 1988 } \\ \text { Calidris alpina } & 53 & 35 & \text { Heyer, 1995 } \\ \text { Calidris minuta } & 29 & 35 & \text { Heyer, 1995 } \\ \text { Sterna fuscata } & 35 & \text { MacMillen } \text { et al., 1977, Flint \& Nagy, } 1984 \\ \text { Thinocorus rumicivorus } & 36.75 & \text { Ehlers \& Morton, 1982 } \\ \text { Bubulcus ibis } & 55.5 & 38 & \text { Ellis, 1980 } \\ \text { Egretta caerulea } & 299.2 & 39 & \text { Ellis, 1980 } \\ \text { Egretta thula } & 290.3 & 40 & \text { Ellis, 1980 } \\ \text { Egretta tricolor } & 314 & 41 & \text { Ellis, 1980 } \\ \text { Colius colius } & 309 & 35 & \text { McKechnie \& Lovegrove, 2001b } \\ \text { Colius striatus } & 40.2 & \text { McKechnie \& Lovegrove, 2001a } \\ \text { Urocolius macrourus } & 51 & & \\ & 51.3 & & \end{array}$

Ins. data

Good

No UCT

No UCT

No UCT

Good

Good

No UCT

No UCT

Good

No UCT

No UCT

Good

Good

No UCT

No UCT

No UCT

Ins. data

No UCT

No UCT

No UCT 


\begin{tabular}{|c|c|c|c|c|}
\hline Leucosarcia melanoleuca & 468 & 36 & McNab, 2000 & No UCT \\
\hline Hemiphaga novaeseelandiae & 435.6 & 30 & McNab, 2000 & Good \\
\hline Geopelia cuneata & 39 & 45 & Schleucher, 1991 & No UCT \\
\hline Geophaps plumifera & 89 & 39 & Withers \& Williams, 1990, Williams et al., 1995 & Good \\
\hline Drepanoptila holosericea & 198 & 33 & Schleucher, 2002 & No UCT \\
\hline Ducula bicolor & 453 & 36 & $\mathrm{McNab}, 2000$ & No UCT \\
\hline Ducula pinon & 583.8 & 37 & $\mathrm{McNab}, 2000$ & No UCT \\
\hline Ducula pistrinaria & 394.2 & 35 & McNab, 2000 & No UCT \\
\hline Ducula radiata & 333.6 & 37 & $\mathrm{McNab}, 2000$ & No UCT \\
\hline Ducula rubricera & 418.8 & 36 & $\mathrm{McNab}, 2000$ & No UCT \\
\hline Ducula rufigaster & 376.7 & 35 & $\mathrm{McNab}, 2000$ & No UCT \\
\hline Ducula zoeae & 456.2 & 32 & $\mathrm{McNab}, 2000$ & Good \\
\hline Gymnophaps albertisii & 241.6 & 36 & $\mathrm{McNab}, 2000$ & Ins. data \\
\hline Ptilinopus melanospilus & 98 & 35 & Schleucher \& Withers, 2002, McNab, 2000 & Ins. data \\
\hline Columba vitiensis & 467.9 & 34 & McNab, 2000 & Ins. data \\
\hline Caloenas nicobarica & 613 & 37 & $\mathrm{McNab}, 2000$ & No UCT \\
\hline Ducula pacifica & 333.4 & 36 & McNab, 2000 & No UCT \\
\hline Goura cristata & 2313.4 & 35 & $\mathrm{McNab}, 2000$ & No UCT \\
\hline Ptilinopus perlatus & 196 & 32 & Schleucher, 2002, McNab, 2000 & No UCT \\
\hline Ptilinopus superbus & 120.4 & 30 & Schleucher, 2002, McNab, 2000 & No UCT \\
\hline Columbina inca & 41.5 & 35 & MacMillen \& Trost, 1967 & No UCT \\
\hline Zenaida macroura & 91.4 & 37.5 & Hudson \& Brush, 1964 & Good \\
\hline Columba livia & 467 & 36.5 & $\mathrm{McNab}, 2000$ & Good \\
\hline Oena capensis & 36 & 40 & Schleucher, 2001 & No UCT \\
\hline Dacelo novaeguineae & 336 & 35 & Buttemer et al., 2003 & No UCT \\
\hline
\end{tabular}




\begin{tabular}{|c|c|c|c|c|}
\hline Bycanistes bucinator & 721 & 30 & Harji, 2003 & ----- \\
\hline Aceros plicatus & 1965 & 35 & McNab, 2001 & No UCT \\
\hline Phoeniculus purpureus & 72.19 & 35 & Boix-Hinzen \& Lovegrove, 1998, Williams et al., 1991 & No UCT \\
\hline Todus mexicanus & 6.3 & 32 & Merola-Zwartjes \& David, 2000 & Good \\
\hline Guira guira & 140 & 33 & Simone, 2003 & ----- \\
\hline Falco rupicoloides & 214 & 33 & Bush et al., 2008 & Ins. data \\
\hline Daptrius ater & 362 & 35 & Wasser, 1986 & Ins. data \\
\hline Leipoa ocellata & 1390 & 37 & Booth, 1989 & Good \\
\hline Callipepla gambelii & 125.5 & 42 & Weathers, 1981, Goldstein \& Nagy, 1985 & Good \\
\hline Coturnix chinensis & 44.9 & 35 & Roberts \& Baudinette, 1986, Prinzinger et al., 1993 & Ins. data \\
\hline Coturnix pectoralis & 95.8 & 35 & Roberts \& Baudinette, 1986 & Ins. data \\
\hline Syrmaticus humiae & 398.83 & 29.2 & Ying et al., 2011 & Good \\
\hline Coturnix japonica & 166 & 36 & Prinzinger \& Hänssler, 1980, Ben-Hamo et al., 2010 & Good \\
\hline Alectoris chukar & 475 & 39 & Marder \& Bernstein, 1983 & Good \\
\hline Coturnix coturnix & 97 & 34 & Weathers, 1981, Ingrid, 1978 & Good \\
\hline Lagopus leucura & 326 & 38 & Johnson, 1968 & Good \\
\hline Lagopus lagopus & 658 & 28 & Mortensen \& Blix, 1986 & No UCT \\
\hline Lagopus muta & 465 & 22 & Mortensen \& Blix, 1986 & No UCT \\
\hline Gallinula mortierii & 954.4 & 35 & McNab \& Ellis, 2006 & Ins. data \\
\hline Gallinula tenebrosa & 512.4 & 36 & McNab \& Ellis, 2006 & No UCT \\
\hline Porphyrio hochstetteri & 2758.3 & 31 & McNab \& Ellis, 2006 & Ins. data \\
\hline Porphyrio porphyrio & 919.3 & 38 & McNab \& Ellis, 2006 & No UCT \\
\hline Gallirallus philippensis & 171.7 & 37 & McNab \& Ellis, 2006 & No UCT \\
\hline Gallinula ventralis & 309.2 & 40 & McNab \& Ellis, 2006 & No UCT \\
\hline Gallirallus australis & 813.5 & 38 & McNab \& Ellis, 2006 & No UCT \\
\hline
\end{tabular}




Porzana cinerea
Megacrex inepta
Gallirallus owstoni
Aramides cajanea
Fulica atra
Mirafra erythrocephala
Alaemon alaudipes
Eremalauda dunni
Eremophila alpestris
Alauda arvensis
Lullula arborea
Bombycilla garrulus
Cardinalis cardinalis
Cardinalis sinuatus
Cnemophilus loriae
Coereba flaveola
Aphelocoma coerulescens
Cyanocitta stelleri
Cyanocitta cristata
Pica nuttalli
Pica pica
Corvus monedula
Corvus corone
Corvus corax
Phytotoma rara

\begin{tabular}{ccl}
47.9 & 37 & McNab \& Ellis, 2006 \\
856.6 & 35 & McNab \& Ellis, 2006 \\
198.8 & 38 & McNab \& Ellis, 2006 \\
374.3 & 38 & McNab \& Ellis, 2006 \\
387 & 34 & Brent et al., 1985 \\
27.3 & 35.1 & Marschall \& Prinzinger, 1991 \\
37.7 & 37.5 & Tieleman et al., 2002 \\
20.6 & 41 & Tieleman et al., 2002 \\
26 & 35 & Trost, 1972 \\
31.7 & 35.1 & Tieleman et al., 2002 \\
25.5 & 40 & Tieleman et al., 2002 \\
64.9 & 27 & Ming et al., 2005 \\
41 & 42.6 & Hinds \& Calder, 1973 \\
32 & 42.8 & Hinds \& Calder, 1973 \\
78.1 & 32 & McNab, 2005 \\
10 & 35 & Merola-Zwartjes, 1998 \\
78.72 & 24 & Mann, 1983 \\
99.96 & 31.8 & Mann, 1983 \\
80.8 & 35 & Misch, 1960 \\
151.9 & 33.5 & Hayworth \& Weathers, 1984 \\
158.9 & 32.5 & Hayworth \& Weathers, 1984 \\
188 & 26 & Daan et al., 1990, Prinzinger, 1976 \\
518 & 26 & Prinzinger, 1976 \\
1203 & 31 & Schwan \& Williams, 1978 \\
41.6 & 30 & Rezende et al., 2001 \\
& & \\
\hline 58 & &
\end{tabular}

Ins. data
No UCT
Ins. data
Ins. data
Good
Good
Good
Good
Good
Ins. data
Ins. data
Ins. data
Good
Good
Ins. data
Ins. data
Ins. data
Ins. data
Ins. data
Good
Good
------
------
No UCT
No UCT 


\begin{tabular}{|c|c|c|c|c|}
\hline Emberiza citrinella & 26.8 & 33 & Wallgren, 1954 & No UCT \\
\hline Emberiza pusilla & 11.3 & 25 & Jin-Song et al., 2001 & Good \\
\hline Emberiza rutila & 15.3 & 32.5 & Jin-Song et al., 2001 & Good \\
\hline Emberiza hortulana & 24.3 & 38 & Wallgren, 1954 & No UCT \\
\hline Amphispiza belli & 18.6 & 37 & Moldenhauer, 1970 & Good \\
\hline Zonotrichia leucophrys & 28.6 & 37 & King, 1964 & Good \\
\hline Emberiza spodocephala & 15.1 & 26 & Ming et al., 2005 & Good \\
\hline Emberiza chrysophrys & 15.94 & 30 & Jin-Song et al., 2005 & Good \\
\hline Lonchura cucullata & 10.6 & 38 & Lovegrove \& Smith, 2003 & No UCT \\
\hline Taeniopygia guttata & 11.7 & 38 & Calder 1964, Marshall \& Prinzinger, 1991 & No UCT \\
\hline Amadina fasciata & 17.2 & 38 & Marschall \& Prinzinger, 1991 & Good \\
\hline Estrilda melpoda & 7.5 & 43 & Marshall \& Prinzinger, 1991 & No UCT \\
\hline Padda oryzivora & 24.9 & 42 & Marshal \& Prinzinger, 1991 & No UCT \\
\hline Estrilda troglodytes & 6.4 & 38 & Cade et al 1965, Weathers \& Nagy, 1984 & Good \\
\hline Lonchura fuscans & 9.5 & 39 & Weathers, 1977 & Good \\
\hline Erythrura gouldiae & 15.5 & 38 & Burton \& Weathers, 2003 & Good \\
\hline Carpodacus mexicanus & 20.4 & 33 & Weathers, 1981, Dawson et al., 1985 & No UCT \\
\hline Carpodacus purpureus & 34 & 35 & Salt, 1952 & Good \\
\hline Carpodacus cassinii & 27.4 & 37 & Weather et al., 1980 & Good \\
\hline Loxia curvirostra & 29.4 & 28.5 & Dawson \& Tordoff, 1964 & No UCT \\
\hline Loxia leucoptera & 29.8 & 22 & Dawson \& Tordoff, 1964 & No UCT \\
\hline Carduelis pinus & 13.8 & 35 & Dawson \& Carey, 1976 & No UCT \\
\hline Carduelis tristis & 12.8 & 35 & Dawson \& Carey, 1976 & Ins. data \\
\hline Carpodacus roseus & 22.5 & 27.5 & Jin-Song et al., 2004 & Ins. data \\
\hline Fringilla montifringilla & 21 & 30 & Jin-Song et al., 2004 & Good \\
\hline
\end{tabular}




$\begin{array}{lccl}\text { Coccothraustes vespertinus } & 60 & 34 & \text { Dawson \& Tordoff, 1959 } \\ \text { Carduelis flammea } & 14.7 & 28 & \text { Reinertsen \& Haftorn, 1986, Pohl \& West, } 1973 \\ \text { Icterus bullockii } & 34 & 35 & \text { Rising, 1969 } \\ \text { Icterus galbula } & 34 & 35 & \text { Rising, 1969 } \\ \text { Lanius collaris } & 43.8 & 35 & \text { Soobramoney } \text { et al., 2003 } \\ \text { Lanius excubitor } & 60 & 36 & \text { Ward \& Pinshow, 1995, Degen } \text { et al., } 1992 \\ \text { Malurus cyaneus } & 8.2 & 35 & \text { Lill } \text { et al., 2006, Weathers \& Stiles, } 1989 \\ \text { Manacus vitellinus } & 15.5 & 32 & \text { Bartholomew } \text { et al., 1983 } \\ \text { Eremiornis carteri } & 12.3 & 39 & \text { Ambrose } \text { et al., 1996 } \\ \text { Lichmera indistincta } & 10.1 & 39 & \text { Vittali } \text { et al., 1999, Collins } \text { et al., 1980 } \\ \text { Lichenostomus virescens } & 25 & 39 & \text { Collins } \text { et al., 1980 } \\ \text { Nectarinia amethystina } & 10 & 30 & \text { Prinzinger } \text { et al., 1989, Seavy, 2006 } \\ \text { Nectarinia bifasciata } & 6.2 & 27 & \text { Prinzinger } \text { et al., 1989, Seavy, 2006 } \\ \text { Anthreptes collaris } & 8.3 & 28 & \text { Prinzinger } \text { et al., 1989 } \\ \text { Nectarinia kilimensis } & 16.2 & 32 & \text { Prinzinger } \text { et al., 1989 } \\ \text { Nectarinia venusta } & 7.6 & 30 & \text { Lübben, 1986, Seavy, 2006 } \\ \text { Nectarinia cuprea } & 7.1 & 35 & \text { Lübben, 1986, Seavy, 2006 } \\ \text { Aethopyga siparaja } & 6.8 & 28 & \text { Prinzinger } \text { et al., 1989, Lübben, 1986 } \\ \text { Nectarinia tacazze } & 13.5 & 31 & \text { Prinzinger } \text { et al., 1989, Lübben, 1986, Seavy, } 2006 \\ \text { Anthreptes } \text { orientalis } & 11.8 & 32 & \text { Prinzinger } \text { et al., 1989 } \\ \text { Nectarinia senegalensis } & 13.7 & 35 & \text { Seavy, 2006 } \\ \text { Aethopyga christinae } & 5.2 & 32 & \text { Prinzinger } \text { et al., 1989 } \\ \text { Paradisaea raggiana } & 215.7 & 33 & \text { McNab, 2005 } \\ \text { Lophorina superba } & 74.6 & 32 & \text { McNab, 2005 } \\ \text { Manucodia keraudrenii } & 170.7 & 29 & \text { McNab, 2005 }\end{array}$

No UCT

No UCT

Good

Good

Ins. data

Good

Good

No UCT

Ins. data

No UCT

Ins. data

No UCT

No UCT

No UCT

No UCT

Ins. data

No UCT

No UCT

No UCT

No UCT

No UCT

No UCT

No UCT

No UCT

No UCT 


Paradisaea rudolphi
Epimachus meyeri
Parotia wahnesi
Manucodia chalybatus
Parotia lawesii
Astrapia stephaniae
Cicinnurus regius
Cicinnurus magnificus
Ptiloris magnificus
Parus atricapillus
Passer domesticus
Syrmaticus ellioti
Pipra mentalis
Pycnonotus sinensis
Actitis hypoleucos
Sitta canadensis
Athene cunicularia
Onychognathus morio
Acridotheres cristatellus
Sturnus sericeus
Onychognathus tristramii
Sturnus vulgaris
Hylophylax naevioides
Sporophila corvina
Cyanerpes cyaneus

$\begin{array}{ccl}156.1 & 33 & \text { McNab, 2005 } \\ 202.7 & 33 & \text { McNab, 2005 } \\ 164.2 & 30 & \text { McNab, 2005 } \\ 177.2 & 30 & \text { McNab, 2005 } \\ 144.9 & 30 & \text { McNab, 2005 } \\ 148.2 & 31 & \text { McNab, 2005 } \\ 54 & 33 & \text { McNab, 2005 } \\ 82.3 & 33 & \text { McNab, 2005 } \\ 179.4 & 31 & \text { McNab, 2005 } \\ 10.3 & 32 & \text { Rising \& Hudson, 1974 } \\ 23 & 35 & \text { Hudson \& Kimzey, 1966 } \\ 388.25 & 31.6 & \text { Ying } \text { et al., 2011 } \\ 12.3 & 32 & \text { Bartholomew et al., 1983 } \\ 25.6 & 33 & \text { Yong-Pu } \text { et al., 2006 } \\ 41.1 & 33 & \text { Heyer, 1995, Tatner \& Bryant, 1993 } \\ 11.2 & 38 & \text { Mugaas \& Templeton, 1970 } \\ 146.7 & 37 & \text { Coulombe, 1970 } \\ 128 & 30 & \text { Chamane \& Downs, 2009 } \\ 117.7 & 32.5 & \text { Lin } \text { et al., 2010 } \\ 64.9 & 34.5 & \text { Yong-Pu } \text { et al., 2006 } \\ 123 & 36.5 & \text { Dmi'el \& Tel-Tzur, 1985 } \\ 75 & 37 & \text { Ricklefs \& Williams, 1984 } \\ 16.1 & 34 & \text { Wiersma } \text { et al., 2007, Steiger } \text { et al., 2009 } \\ 11 & 39.2 & \text { Weathers, 1997 } \\ 13.5 & 35 & \text { Wiersma } \text { et al., 2007, Mata, 2010 } \\ & & \end{array}$

No UCT

No UCT

No UCT

Ins. data

No UCT

No UCT

No UCT

No UCT

No UCT

Ins. data

Good

Good

No UCT

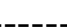

Ins. data

Good

No UCT

Ins. data

Good

Ins. data 


\begin{tabular}{|c|c|c|c|c|}
\hline Thryothorus ludovicianus & 14.9 & 35 & Eberhardt, 1994 & Ins. data \\
\hline Zosterops lateralis & 11.8 & 36.5 & Maddocks \& Gieser, 1997, Weathers \& Sttiles, 1989 & No UCT \\
\hline Zosterops erythropleurus & 9.16 & 27.5 & Jin-Song et al., 2005 & Good \\
\hline Anhinga anhinga & 1040 & 40 & Hennemann, 1983 & No UCT \\
\hline Phalacrocorax auritus & 1330 & 40 & Hennemann, 1983, Enstipp et al., 2006 & No UCT \\
\hline Trachyphonus darnaudii & 36.6 & 38 & $\mathrm{McNab}, 2001$ & No UCT \\
\hline Ramphastos toco & 582 & 34 & McNab, 2001 & No UCT \\
\hline Ramphastos dicolorus & 328.9 & 34 & McNab, 2001 & No UCT \\
\hline Selenidera maculirostris & 130.5 & 34 & $\mathrm{McNab}, 2001$ & No UCT \\
\hline Pteroglossus bailloni & 133 & 34 & $\mathrm{McNab}, 2001$ & No UCT \\
\hline Pteroglossus aracari & 200.7 & 35 & McNab, 2001 & No UCT \\
\hline Ramphastos tucanus & 420.3 & 35 & $\mathrm{McNab}, 2001$ & No UCT \\
\hline Aulacorhynchus sulcatus & 131.7 & 31 & $\mathrm{McNab}, 2001$ & Ins. data \\
\hline Aulacorhynchus prasinus & 174.7 & 34 & $\mathrm{McNab}, 2001$ & No UCT \\
\hline Podiceps nigricollis & 317 & 38 & Ellis \& Jehl, 2003 & No UCT \\
\hline Puffinus pacificus & 367 & 34 & Whittow et al., 1987 & ------ \\
\hline Fulmarus glacialis & 651 & 22 & Gabrielsen et al., 1988 & No UCT \\
\hline Cacatua roseicapilla & 271 & 33 & Dawson \& Fisher, 1982, Williams et al., 1991 & Good \\
\hline Nestor meridionalis & 369.3 & 26 & McNab \& Salisbury, 1995 & No UCT \\
\hline Nestor notabilis & 836.9 & 28 & McNab \& Salisbury, 1995 & Ins. data \\
\hline Cyanoramphus unicolor & 129.4 & 35 & McNab \& Salisbury, 1995 & No UCT \\
\hline Cyanoramphus auriceps & 52.9 & 37 & McNab \& Salisbury, 1995 & No UCT \\
\hline Cyanoramphus novaezelandiae & 56.1 & 37 & McNab \& Salisbury, 1995 & No UCT \\
\hline Neophema pulchella & 40 & 32 & Prinzinger \& Hänssler, 1980, Burton et al., 2008 & Ins. data \\
\hline Neophema splendida & 40.86 & 29 & Burton et al., 2008 & Ins. data \\
\hline
\end{tabular}




\begin{tabular}{|c|c|c|c|}
\hline Neophema elegans & 42.02 & 32 & Burton et al., 2008 \\
\hline Melopsittacus undulatus & 33.7 & 41 & $\begin{array}{l}\text { Weathers \& Schoenbaechler, 1976, Buttemer et al., } \\
1986\end{array}$ \\
\hline Agapornis nigrigenis & 41.37 & 35 & Burton et al., 2008 \\
\hline Agapornis roseicollis & 53.53 & 30.5 & Burton et al., 2008 \\
\hline Agapornis personatus & 46.77 & 34 & Burton et al., 2008 \\
\hline Bolborhynchus lineola & 55.7 & 32 & Bucher, 1980 \\
\hline Agapornis fischeri & 56.7 & 34 & Burton et al., 2008 \\
\hline Amazona viridigenalis & 341 & 35 & Bucher, 1985 \\
\hline Myiopsitta monachus & 80.4 & 38.5 & Weathers \& Caccamise, 1975 \\
\hline Nothoprocta perdicaria & 458 & 28 & Withers et al., 1987 \\
\hline Pterocles alchata & 242 & 32.1 & Hinsley et al., 1993 \\
\hline Pterocles orientalis & 386.4 & 35.9 & Hinsley et al., 1993 \\
\hline Aptenodytes patagonicus & 11080 & 10 & Froget et al., 2002 \\
\hline Eudyptula minor & 1059.3 & 25 & Richman \& Lovvorn, 2011 \\
\hline Spheniscus humboldti & 3870 & 30 & Drent \& Stonehouse, 1971 \\
\hline Otus leucotis & 221.1 & 32 & Smit et al., 2008 \\
\hline Micrathene whitneyi & 45 & 38 & Ligon, 1968 \\
\hline Bubo virginianus & 1000 & 32.2 & Ganey et al., 1993 \\
\hline Strix occidentalis & 571 & 25.2 & Ganey et al., 1993, Weathers et al., 2001 \\
\hline Glaucidium gnoma & 52 & 34 & Ligon, 1968 \\
\hline Megascops asio & 141.5 & 34 & Ligon, 1969 \\
\hline Megascops trichopsis & 119 & 35 & Ligon, 1969 \\
\hline Aegolius acadicus & 118.1 & 33 & Ligon, 1969 \\
\hline Strix aluco & 419 & 35 & Bech \& Præsteng, 2004 \\
\hline Aegolius funereus & 130 & 25 & Hohtola, 1994 \\
\hline
\end{tabular}

Ins. data

Good

No UCT

No UCT

No UCT

Ins. data

No UCT

No UCT

Good

No UCT

Good

Good

No UCT

Ins. data

Ins. data

Ins. data

Good

Good

Good

No UCT

Good

No UCT

Good

No UCT

No UCT 


$\begin{array}{lccl}\text { Bubo scandiaca } & 2026 & 18.5 & \text { Gessaman, 1972 } \\ \text { Tyto alba } & 533.2 & 32.5 & \text { Edwards, 1987 } \\ \text { Apteryx australis } & 3137 & 30 & \text { McNab, 1996 } \\ \text { Apteryx haastii } & 2529 & 30 & \text { McNab, 1996 } \\ \text { Apteryx owenii } & 1377 & 29 & \text { McNab, 1996 } \\ \text { Patagona gigas } & 19.1 & 37 & \text { Lasiewski } \text { et al., 1967 } \\ \text { Eugenes fulgens } & 6.6 & 37 & \text { Lasiewski \& Lasiewski, } 1967 \\ \text { Archilochus alexandri } & 3.3 & 35 & \text { Lasiewski, 1963 } \\ \text { Calypte anna } & 4.5 & 37 & \text { Lasiewski, 1963 } \\ \text { Calypte costae } & 3.2 & 37 & \text { Lasiewski, 1963 } \\ \text { Selasphorus rufus } & 3.8 & 37 & \text { Lasiewski, 1963 } \\ \text { Selasphorus sasin } & 3.7 & 36 & \text { Lasiewski, 1963 } \\ \text { Stellula calliope } & 3 & 38 & \text { Lasiewski, 1963 } \\ \text { Turnix suscitator } & 58.1 & 39 & \text { Prinzinger } \text { et al., 1993 } \\ & & & \\ \text { Mammals } & & & \\ \text { Setifer setosus } & 530 & 33 & \text { McNab, 1980 } \\ \text { Aotus trivirgatus } & 1020 & 30 & \text { Le-Maho } \text { et al., 1981 } \\ \text { Capra hircus } & 21000 & 30 & \text { Holmes \& Moore, 1981; Luiting } \text { et al., } 1985 \\ \text { Kobus ellipsiprymnus } & 37 & \text { Taylor } \text { et al., 1969 } \\ \text { Madoqua kirkii } & 1.00 \mathrm{E}+05 & 35 & \text { Maskrey \& Hoppe, 1979 } \\ \text { Philantomba monticola } & 4600 & 32 & \text { Haim \& Skinner, 1991 } \\ \text { Raphicerus campestris } & 4400 & 33 & \text { Haim \& Skinner, 1991 } \\ \text { Sus scrofa } & 8500 & \text { Huynh } \text { et al., 2005 } \\ \text { Pecari tajacu } & 48000 & \text { Zervanos, 1975 } \\ & 20000 & 35 & \end{array}$

No UCT

Good

No UCT

No UCT

No UCT

No UCT

No UCT

No UCT

No UCT

No UCT

No UCT

No UCT

No UCT

No UCT

No UCT

Good

No UCT

No UCT

No UCT

No UCT

No UCT

Good 


\begin{tabular}{|c|c|c|c|c|}
\hline Tragulus javanicus & 1618 & 29 & Whittow et al., 1977 & Good \\
\hline Canis mesomelas & 7720 & 26 & Downs et al., 1991 & Ins. data \\
\hline Canis latrans & 10000 & 26 & Golightly \& Ohmart, 1983 & Good \\
\hline Vulpes macrotis & 1868 & 33 & Golightly \& Ohmart, 1983 & Good \\
\hline Cerdocyon thous & 5444 & 37 & Henneman et al., 1983 & No UCT \\
\hline Canis lupus & 18066.67 & 32.5 & Irving et al., 1995 & No UCT \\
\hline Vulpes vulpes & 5010 & 30 & Irving et al., 1995 & No UCT \\
\hline Vulpes zerda & 1106 & 32 & Noll-Banholzer, 1979 & Good \\
\hline Fossa fossana & 2260 & 34 & McNab, 1995 & Ins. data \\
\hline Leptailurus serval & 10100 & 30 & Downs et al., 1991 & No UCT \\
\hline Galerella sanguinea & 540 & 31 & Kamau et al., 1979 & Ins. data \\
\hline Suricata suricatta & 850 & 33 & Müller \& Lojewski, 1986 & Good \\
\hline Spilogale putorius & 624 & 36 & Knudsen \& Kilgore, 1990 & Good \\
\hline Eira barbara & 2950 & 35 & McNab, 1995 & No UCT \\
\hline Martes americana & 1038 & 35 & Worthen \& Kilgore, 1981 & No UCT \\
\hline Nasua nasua & 4000 & 33 & Chevillard-Hugot et al., 1979 & Good \\
\hline Nasua narica & 5554 & 35 & Chevillard-Hugot et al., 1979 & ------ \\
\hline Potos flavus & 2400 & 30 & Müller et al., 1983 & Good \\
\hline Ailurus fulgens & 5740 & 36 & McNab, 1988 & Ins. data \\
\hline Genetta tigrina & 1732 & 35 & Willard et al., 1980 & No UCT \\
\hline Nandinia binotata & 4270 & 34 & McNab, 1995 & Ins. data \\
\hline Arctictis binturong & 14280 & 36 & McNab, 1995 & No UCT \\
\hline Arctogalidia trivirgata & 2010 & 38 & McNab, 1995 & No UCT \\
\hline Paradoxurus hermaphroditus & 3160 & 36 & McNab, 1995 & No UCT \\
\hline Saccopteryx bilineata & 8.2 & 35 & Genoud \& Bonaccorso, 1986 & Ins. data \\
\hline
\end{tabular}




$\begin{array}{lccl}\text { Peropteryx macrotis } & 5.1 & 37 & \text { Genoud } \text { et al., 1990 } \\ \text { Rhinonicteris aurantia } & 8.27 & 37.5 & \text { Baudinette } \text { et al., 2000 } \\ \text { Hipposideros maggietaylorae } & 18.2 & 34 & \text { Bonaccorso \& McNab, 2003 } \\ \text { Hipposideros diadema } & 37.2 & 34 & \text { Bonaccorso \& McNab, 2003 } \\ \text { Hipposideros galeritus } & 8.5 & 32 & \text { McNab, 1989 } \\ \text { Macroderma gigas } & 107 & 35 & \text { Baudinette } \text { et al., 2000, Leitner \& Nelson, } 1966 \\ \text { Molossus molossus } & 15.6 & 36 & \text { McNab, 1969 } \\ \text { Tadarida brasiliensis } & 16.9 & 32.7 & \text { Soriano } \text { et al., 2002 } \\ \text { Mormoops megalophylla } & 16.5 & 39.5 & \text { Bonaccorso } \text { et al., 1992 } \\ \text { Pteronotus davyi } & 9.4 & 43 & \text { Bonaccorso } \text { et al., 1992 } \\ \text { Pteronotus parnellii } & 19.2 & 40.5 & \text { Bonaccorso } \text { et al., 1992 } \\ \text { Pteronotus personatus } & 14 & 38.5 & \text { Bonaccorso } \text { et al., 1992 } \\ \text { Chrotopterus auritus } & 96.1 & 34 & \text { McNab, 1969 } \\ \text { Pteronotus quadridens } & 4.9 & 38 & \text { Rodríguez-Durán, 1995 } \\ \text { Mormoops blainvillei } & 8.6 & 36 & \text { Rodríguez-Durán, 1995 } \\ \text { Natalus tumidirostris } & 5.4 & 35 & \text { Genoud } \text { et al., 1990 } \\ \text { Diaemus youngi } & 36.6 & 30 & \text { McNab, 1969 } \\ \text { Desmodus rotundus } & 29.4 & 37.5 & \text { McNab, 1969 } \\ \text { Noctilio albiventris } & 27 & 38 & \text { McNab, 1969 } \\ \text { Noctilio leporinus } & 61 & 38 & \text { McNab, 1969 } \\ \text { Glossophaga longirostris } & 13.5 & 36 & \text { Arends } \text { et al., 1995 } \\ \text { Leptonycteris yerbabuenae } & 44 & 38 & \text { Arends } \text { et al., 1995 } \\ \text { Leptonycteris curasoae } & 24 & 35 & \text { Arends } \text { et al., 1995 } \\ \text { Glossophaga soricina } & 9.6 & 35.2 & \text { Cruz-Neto \& Abe, 1997 } \\ \text { Phyllostomus elongatus } & 35.6 & 36 & \text { McNab, 1969 }\end{array}$

No UCT

Ins. data

No UCT

No UCT

Ins. data

Good

Ins. data

Good

No UCT

No UCT

No UCT

No UCT

No UCT

Good

No UCT

Ins. data

No UCT

No UCT

No UCT

Good

No UCT

No UCT

No UCT

Good

No UCT 


$\begin{array}{lccl}\text { Rhinophylla pumilio } & 9.5 & 35 & \text { McNab, 1969 } \\ \text { Uroderma bilobatum } & 16.2 & 35 & \text { McNab, 1969 } \\ \text { Tonatia bidens } & 27.4 & 35 & \text { McNab, 1969 } \\ \text { Artibeus concolor } & 19.7 & 38 & \text { McNab, 1969 } \\ \text { Phyllostomus hastatus } & 84.2 & 35 & \text { McNab, 1969 } \\ \text { Artibeus lituratus } & 70.1 & 36 & \text { McNab, 1969 } \\ \text { Phyllostomus discolor } & 33.5 & 37 & \text { McNab, 1969 } \\ \text { Carollia perspicillata } & 14.9 & 35 & \text { McNab, 1969 } \\ \text { Artibeus jamaicensis } & 45.2 & 35 & \text { McNab, 1969 } \\ \text { Sturnira lilium } & 21.9 & 35.5 & \text { McNab, 1969 } \\ \text { Diphylla ecaudata } & 27.8 & 30 & \text { McNab, 1969 } \\ \text { Anoura caudifer } & 11.5 & 35 & \text { McNab, 1969 } \\ \text { Choeroniscus periosus } & 15 & 37.7 & \text { McNab, 1969 } \\ \text { Monophyllus redmani } & 8.7 & 35 & \text { Rodríguez-Durán, 1995 } \\ \text { Erophylla bombifrons } & 16.1 & 33 & \text { Rodríguez-Durán, 1995 } \\ \text { Anoura latidens } & 13.6 & 36.2 & \text { Soriano } \text { et al., 2002 } \\ \text { Sturnira erythromos } & 15.9 & 32.5 & \text { Soriano } \text { et al., 2002 } \\ \text { Macroglossus minimus } & 16.3 & 33 & \text { Bartels } \text { et al., 1998 } \\ \text { Pteropus scapulatus } & 440 & 35 & \text { Bartholomew et al., 1964 } \\ \text { Pteropus poliocephalus } & 825 & 35 & \text { Bartholomew et al., 1964 } \\ \text { Dobsonia minor } & 73.7 & 35 & \text { Bartholomew et al., 1970 } \\ \text { Melonycteris melanops } & 53.3 & 33 & \text { Bonaccorso \& McNab, } 1997 \\ \text { Syconycteris australis } & 17.8 & 33 & \text { Geiser } \text { et al., 1996 } \\ \text { Eonycteris spelaea } & 51.6 & 32.5 & \text { McNab, 1989 } \\ \text { Cynopterus brachyotis } & 37.4 & 37 & \text { McNab, 1989 }\end{array}$

\author{
Ins. data \\ No UCT \\ Ins. data \\ No UCT \\ Good \\ Good \\ Good \\ Good \\ Good \\ No UCT \\ Good \\ Ins. data \\ No UCT \\ No UCT \\ No UCT \\ Good \\ No UCT \\ Good \\ No UCT \\ No UCT \\ No UCT \\ No UCT \\ Ins. data \\ Good
}




$\begin{array}{lccl}\text { Pteropus rodricensis } & 254.5 & 35.5 & \text { McNab \& Bonaccorso, 2001 } \\ \text { Dobsonia praedatrix } & 179.5 & 33 & \text { McNab \& Bonaccorso, 2001 } \\ \text { Dobsonia anderseni } & 241.4 & 34 & \text { McNab \& Bonaccorso, 2001 } \\ \text { Nyctimene albiventer } & 30.9 & 34 & \text { McNab \& Bonaccorso, 2001 } \\ \text { Histiotus velatus } & 11.2 & 31 & \text { McNab, 1969 } \\ \text { Miniopterus schreibersii } & 10.91 & 37.5 & \text { Baudinette } \text { et al., 2000 } \\ \text { Lasiurus cinereus } & 27.5 & 34 & \text { Cryan \& Wolf, 2003 } \\ \text { Myotis thysanodes } & 6 & 36.5 & \text { O'Farrell \& Studier, 1970 } \\ \text { Nyctophilus gouldi } & 10 & 32.5 & \text { Geiser \& Brigham, 2000 } \\ \text { Nyctophilus geoffroyi } & 7 & 33.2 & \text { Geiser \& Brigham, 2000 } \\ \text { Chalinolobus gouldii } & 17.5 & 40 & \text { Hosken \& Withers, 1997 } \\ \text { Myotis keaysi } & 5 & 33.4 & \text { Machado \& Soriano, 2007 } \\ \text { Myotis oxyotus } & 4.8 & 32.5 & \text { Machado \& Soriano, 2007 } \\ \text { Myotis lucifugus } & 9.5 & 34.5 & \text { Machado \& Soriano, 2007 } \\ \text { Vespadelus vulturnus } & 4 & 33.3 & \text { Willis } \text { et al., 2005 } \\ \text { Chaetophractus nationi } & 2150 & 35 & \text { McNab, 1980 } \\ \text { Chaetophractus vellerosus } & 1110 & 35 & \text { McNab, 1980 } \\ \text { Dasypus novemcinctus } & 3320 & 38 & \text { McNab, 1980 } \\ \text { Euphractus sexcinctus } & 8190 & 37 & \text { McNab, 1980 } \\ \text { Tolypeutes matacus } & 1160 & 36 & \text { McNab, 1980 } \\ \text { Zaedyus pichiy } & 1740 & 35 & \text { McNab, 1980 } \\ \text { Dasycercus cristicauda } & 86 & 37 & \text { Kennedy \& Macfarlane, 1971 } \\ \text { Ningaui yvonnae } & 11.6 & 35 & \text { Geiser \& Baudinette, 1988 } \\ \text { Planigale gilesi } & 9.4 & 33 & \text { Geiser \& Baudinette, 1988 } \\ \text { Planigale maculata } & 13.1 & 35 & \text { Morton \& Lee, 1978 }\end{array}$

No UCT

Ins. data No UCT Ins. data Ins. data Good

Good

No UCT

No UCT

No UCT

No UCT

No UCT

Ins. data

Good

Ins. data

Ins. data

No UCT

No UCT

No UCT

No UCT

Good

No UCT

No UCT

No UCT 


$\begin{array}{lccl}\text { Sminthopsis crassicaudata } & 19 & 38 & \text { Geiser \& Baudinette, 1988 } \\ \text { Sminthopsis macroura } & 24.8 & 34 & \text { Song } \text { et al., 1995 } \\ \text { Caluromys derbianus } & 357 & 35 & \text { McNab, 1978 } \\ \text { Chironectes minimus } & 946 & 34 & \text { McNab, 1978 } \\ \text { Didelphis marsupialis } & 1329 & 36 & \text { McNab, 1978 } \\ \text { Didelphis virginiana } & 3257 & 34 & \text { McNab, 1978 } \\ \text { Lutreolina crassicaudata } & 812 & 35 & \text { McNab, 1978 } \\ \text { Marmosa lepida } & 106 & 35 & \text { Nelson \& Yousaf, 1979 } \\ \text { Marmosa robinsoni } & 122 & 36 & \text { McNab, 1978 } \\ \text { Metachirus nudicaudatus } & 336 & 36 & \text { McNab, 1978 } \\ \text { Monodelphis brevicaudata } & 111 & 36 & \text { McNab, 1978 } \\ \text { Philander opossum } & 751 & 36 & \text { McNab, 1978 } \\ \text { Thylamys elegans } & 40.2 & 35 & \text { Bozinovic } \text { et al., 2005 } \\ \text { Acrobates pygmaeus } & 14 & 35.1 & \text { Fleming, 1985 } \\ \text { Cercartetus lepidus } & 12 & 33 & \text { Geiser, 1987 } \\ \text { Cercartetus concinnus } & 18.6 & 30 & \text { Geiser, 1987 } \\ \text { Lagorchestes conspicillatus } & 2660 & 35 & \text { Dawson \& Bennett, 1978 } \\ \text { Macropus giganteus } & 26200 & 35 & \text { Dawson \& Hulbert, 1970; Dawson } \text { et al., 2000 } \\ \text { Macropus rufus } & 32490 & 35 & \text { Dawson \& Hulbert, 1970; Dawson } \text { et al., 2000 } \\ \text { Setonix brachyurus } & 2674 & 32.5 & \text { Kinnear \& Shield, 1975 } \\ \text { Dendrolagus matschiei } & 6960 & 37 & \text { McNab, 1988 } \\ \text { Petaurus breviceps } & 128.1 & 31 & \text { Fleming, 1980; Dawson \& Hulbert, 1970 } \\ \text { Phalanger carmelitae } & 1389.7 & 32.5 & \text { McNab, 2008 } \\ \text { Spilocuscus maculatus } & 4250 & 35.4 & \text { Dawson \& Degabriele, 1973 } \\ \text { Phalanger sericeus } & 1353.2 & 30 & \text { McNab, 2008 } \\ \end{array}$

No UCT

Ins. data No UCT No UCT

Ins. data

No UCT

Good

Ins. data

No UCT

No UCT

Ins. data

Good

Ins. data

Good

No UCT

Good

Ins. data

Ins. data

Good

Ins. data

Good

No UCT

No UCT

No UCT 


$\begin{array}{lccl}\text { Phascolarctos cinereus } & 4765 & 26 & \text { Degabriele \& Dawson, } 1979 \\ \text { Potorous tridactylus } & 1120 & 30 & \text { Nicol, 1976 } \\ \text { Bettongia gaimardi } & 1700 & 20 & \text { Rose, 1997 } \\ \text { Cercartetus nanus } & 70 & 35 & \text { Bartholomew \& MacMillen, 1961 } \\ \text { Pseudocheirus peregrinus } & 872 & 32.5 & \text { Kinnear \& Shield, 1975 } \\ \text { Cercopithecus mitis } & 8500 & 28 & \text { Müller } \text { et al., 1983 } \\ \text { Petauroides volans } & 1141 & 25 & \text { Rübsamen } \text { et al., 1984 } \\ \text { Tarsipes rostratus } & 10 & 34 & \text { Withers } \text { et al., 1990 } \\ \text { Lasiorhinus latifrons } & 25000 & 39 & \text { Wells, 1978 } \\ \text { Atelerix albiventris } & 450 & 35 & \text { McNab, 1980 } \\ \text { Erinaceus concolor } & 822.7 & 31.5 & \text { Krol, 1994 } \\ \text { Crocidura russula } & 10.4 & 35 & \text { Sparti, 1990 } \\ \text { Crocidura suaveolens } & 6.5 & 35 & \text { Sparti, 1990 } \\ \text { Heterohyrax brucei } & 2000 & 35 & \text { Bartholomew \& Rainey, } 1971 \\ \text { Procavia capensis } & 2400 & 35 & \text { Rübsamen } \text { et al., 1979 } \\ \text { Brachylagus idahoensis } & 432 & 25.5 & \text { Katzner } \text { et al., 1997 } \\ \text { Lepus alleni } & 3362 & 35 & \text { Dawson \& Schmidt-Nielsen, } 1966 \\ \text { Sylvilagus audubonii } & 672.4 & 40 & \text { Hinds, 1973 } \\ \text { Elephantulus edwardii } & 49.8 & 36 & \text { Leon } \text { et al., 1983 } \\ \text { Macroscelides proboscideus } & 45 & 38 & \text { Roxburg \& Perrin, 1994 } \\ \text { Zaglossus bruijni } & 10300 & 33 & \text { McNab, 1984 } \\ \text { Isoodon obesulus } & 1020 & 35 & \text { Larcombe, 2002 } \\ \text { Macrotis lagotis } & 1011 & 35 & \text { Kinnear \& Shield, 1975 } \\ \text { Tamandua tetradactyla } & 3500 & 35 & \text { McNab, 1984 } \\ \text { Callithrix pygmaea } & 153 & 34 & \text { Genoud } \text { et al., 1997 } \\ & & & \end{array}$

Good Ins. data Good Good Good Good

Good

No UCT

Good

No UCT

Good

Good

Good

No UCT

Good

Good

Good

Good

Good

No UCT

No UCT

No UCT

Good

No UCT

No UCT 


$\begin{array}{lcc}\text { Saimiri sciureus } & 850 & 35 \\ \text { Colobus guereza } & 10500 & 28 \\ \text { Macaca fuscata } & 9550 & 35 \\ \text { Microcebus murinus } & 105 & 28 \\ \text { Eulemur fulvus } & 2330 & 40 \\ \text { Perodicticus potto } & 1090 & 29 \\ \text { Nycticebus coucang } & 1300 & 33 \\ \text { Nycticebus pygmaeus } & 388 & 35 \\ \text { Tarsius syrichta } & 125 & 35 \\ \text { Aplodontia rufa } & 630 & 35 \\ \text { Cryptomys hottentotus } & 102 & 32 \\ \text { Cryptomys bocagei } & 94 & 32.5 \\ \text { Cryptomys mechowi } & 267 & 30 \\ \text { Cryptomys damarensis } & 124 & 31 \\ \text { Georychus capensis } & 192.6 & 34 \\ \text { Heterocephalus glaber } & 39.2 & 37 \\ \text { Dolichotis salinicola } & 1613 & 37 \\ \text { Kerodon rupestris } & 801 & 36 \\ \text { Hydrochoerus hydrochaeris } & 26400 & 33 \\ \text { Microtus guentheri } & 51.3 & 34 \\ \text { Microtus californicus } & 44 & 40 \\ \text { Microtus longicaudus } & 26.9 & 33.5 \\ \text { Phyllotis darwini } & 59 & 34 \\ \text { Loxodontomys micropus } & 62.3 & 33 \\ \text { Peromyscus maniculatus } & 17 & 37\end{array}$

Stitt \& Hardy, 1971; Malinow \& Wagner, 1966

Müller et al., 1983

Nakayama et al., 1971

Aujard et al., 1998

Daniel, 1984

Hildwein \& Goffart, 1975

Müller, 1979

Xiao et al., 2010

McNab \& Wright, 1987

McNab, 1979

Bennett et al., 1994

Bennett et al., 1994

Bennett et al., 1994

Lovegrove, 1986

Lovvegrove, 1987

McNab, 1966

Arends \& McNab, 2001

Arends \& McNab, 2001

Arends \& McNab, 2001

Banin et al., 1990, Haim \& Izhaki, 1995

Bell et al., 1986

Bell et al., 1986

Bozinovic \& Rosenmann, 1988

Bozinovic \& Rosenmann, 1988

Brower \& Cade, 1966
Ins. data

Good

No UCT

No UCT

No UCT

Ins. data

Good

Ins. data

Ins. data

No UCT

Ins. data

Ins. data

Ins. data

Good

Good

Ins. data

Ins. data

No UCT

No UCT

No UCT

Good

Good

Good

Ins. data

Ins. data 


\begin{tabular}{lccl} 
Megadontomys thomasi & 111 & 35 & Buffenstein \& Jarvis, 1985 \\
Myodes gapperi & 20.8 & 34 & Deavers \& Hudson, 1981 \\
Peromyscus leucopus & 21.2 & 34.5 & Deavers \& Hudson, 1981 \\
Isthmomys pirrensis & 140.4 & 34 & Hill, 1975 \\
Microtus arvalis & 24 & 30 & Jansky, 1959 \\
Ochrotomys nuttalli & 19.5 & 36 & Layne \& Dolan, 1975 \\
Peromyscus gossypinus & 21.5 & 35.3 & Layne \& Dolan, 1975 \\
Neotoma lepida & 106 & 35 & McNab, 1966 \\
Oxymycterus roberti & 83.5 & 34 & McNab, 1984 \\
Neofiber alleni & 258.1 & 34 & McNab, 1992 \\
Ondatra zibethicus & 1004.6 & 30 & McNab, 1992 \\
Microtus pinetorum & 23.8 & 35 & McNab, 1992 \\
Lemmiscus curtatus & 30.3 & 33 & McNab, 1998 \\
Microtus richardsoni & 51.3 & 33.5 & McNab, 1998 \\
Peromyscus californicus & 49.6 & 34.5 & McNab \& Morrison, 1963 \\
Peromyscus truei & 33 & 33 & McNab \& Morrison, 1963 \\
Peromyscus crinitus & 20.9 & 35 & McNab \& Morrison, 1963 \\
Peromyscus eremicus & 21.5 & 36.5 & McNab \& Morrison, 1963 \\
Microtus montanus & 51.2 & 31 & Packard, 1968 \\
Reithrodontomys megalotis & 7.6 & 35 & Pearson, 1960 \\
Myopus schisticolor & 26.4 & 24 & Saarela \& Hissa, 1993 \\
Cricetulus barabensis & 31.4 & 30 & Song \& Wang, 2003 \\
Graomys griseoflavus & 69.4 & 34 & Caviedes-Vidal et al., 1987 \\
Phodopus sungorus & 24 & 35 & Weiner \& Heldmaier, 1987 \\
Onychomys torridus & 19.1 & 35 & Whitford \& Conley, 1971 \\
& & & \\
\hline & & &
\end{tabular}

Good

Ins. data Ins. data No UCT

Ins. data

No UCT

Ins. data Ins. data Ins. data

Good

Good

Good

Ins. data No UCT No UCT No UCT Ins. data No UCT No UCT Good 


\begin{tabular}{|c|c|c|c|c|}
\hline Microtus pennsylvanicus & 38.9 & 29 & Wiegert, 1961 & Ins. data \\
\hline Microtus ochrogaster & 47.4 & 34 & Wunder et al., 1977 & No UCT \\
\hline Ctenomys talarum & 116 & 30 & Busch, 1989 & No UCT \\
\hline Priodontes maximus & 45190 & 34 & McNab, 1980 & No UCT \\
\hline Cabassous centralis & 3810 & 32.5 & McNab, 1980 & Good \\
\hline Dasyprocta azarae & 3849 & 35 & Arends \& McNab, 2001 & No UCT \\
\hline Myoprocta acouchy & 914 & 30 & Arends \& McNab, 2001 & Ins. data \\
\hline Dasyprocta leporina & 2687 & 34 & Arends \& McNab, 2001 & No UCT \\
\hline Napaeozapus insignis & 20.9 & 34 & Brower \& Cade, 1966 & Ins. data \\
\hline Jaculus jaculus & 74.5 & 35 & Hooper \& Hilali, 1972 & Good \\
\hline Jaculus orientalis & 139.1 & 33 & Hooper \& Hilali, 1972 & Good \\
\hline Thrichomys apereoides & 323 & 35 & Arends \& McNab, 2001 & No UCT \\
\hline Erethizon dorsatum & 7710 & 20 & Irving et al., 1995 & No UCT \\
\hline Geomys bursarius & 197 & 33 & Bradley \& Yousaf, 1975 & No UCT \\
\hline Thomomys umbrinus & 85 & 35 & Bradley et al., 1974 & Good \\
\hline Thomomys talpoides & 106 & 32 & Bradley et al., 1974 & Good \\
\hline Geomys pinetis & 202 & 35 & McNab, 1966 & Ins. data \\
\hline Thomomys bottae & 143 & 30 & Vleck, 1979 & Good \\
\hline Chaetodipus intermedius & 15.2 & 36 & Bradley et al., 1975 & Good \\
\hline Dipodomys microps & 57.2 & 32 & Breyen et al., 1973 & Good \\
\hline Chaetodipus penicillatus & 16 & 37 & Brower \& Cade, 1966 & No UCT \\
\hline Dipodomys merriami & 34.7 & 35 & Dawson, 1955 & No UCT \\
\hline Dipodomys panamintinus & 64.2 & 34 & Dawson, 1955 & No UCT \\
\hline Liomys salvini & 43.8 & 34 & Hudson \& Rummel, 1966 & No UCT \\
\hline Liomys irroratus & 48.1 & 34 & Hudson \& Rummel, 1966 & No UCT \\
\hline
\end{tabular}




\begin{tabular}{|c|c|c|c|}
\hline Heteromys anomalus & 69.3 & 33 & McNab, 1979 \\
\hline Dipodomys deserti & 106 & 35 & McNab, 1979 \\
\hline Chaetodipus hispidus & 35.8 & 33.5 & Wang \& Hudson, 1970 \\
\hline Hystrix africaeaustralis & 10700 & 27 & Haim et al., 1990 \\
\hline Abrothrix longipilis & 42.3 & 32 & Bozinovic \& Rosenmann, 1988 \\
\hline Abrothrix andinus & 34.6 & 34 & Bozinovic \& Rosenmann, 1988 \\
\hline Auliscomys boliviensis & 76.8 & 31 & Bozinovic \& Rosenmann, 1988 \\
\hline Micaelamys namaquensis & 64.2 & 34.49 & Buffenstein, 1984 \\
\hline Gerbillurus paeba & 33.9 & 35.1 & Buffenstein, 1984 \\
\hline Gerbillus pusillus & 12.6 & 38 & Buffenstein \& Jarvis, 1985 \\
\hline Mus musculus & 46 & 33 & Buffenstein \& Jarvis, 1985 \\
\hline Rattus fuscipes & 76 & 32.9 & Collins, 1973 \\
\hline Rattus villosissimus & 250.6 & 35 & Collins \& Brdshaw, 1973 \\
\hline Akodon azarae & 23.5 & 32 & Dalby \& Heath, 1976 \\
\hline Gerbillurus setzeri & 46.1 & 34.8 & Dempster et al.,1998 \\
\hline Gerbillurus vallinus & 38.8 & 35 & Dempster et al.,1999 \\
\hline Gerbillurus tytonis & 29.9 & 34.9 & Downs \& Perrin, 1990 \\
\hline Mystromys albicaudatus & 93.78 & 30 & Downs \& Perrin, 1995 \\
\hline Gerbillus andersoni & 35.8 & 35 & Haim, 1984 \\
\hline Gerbillus nanus & 28.2 & 34 & Haim, 1984 \\
\hline Dipodillus dasyurus & 27.6 & 35 & Haim, 1987 \\
\hline Otomys irroratus & 111.6 & 28 & Haim \& Fairall, 1987 \\
\hline Apodemus mystacinus & 42.3 & 32 & Haim et al., 1993 \\
\hline Mus macedonicus & 14.5 & 33 & Haim et al., 1999 \\
\hline Baiomys taylori & 7.3 & 36 & Hudson, 1965 \\
\hline
\end{tabular}

No UCT

Ins. data

Ins. data

Good

Good

Good

No UCT

Good

Good

Good

Ins. data

Ins. data

Good

Ins. data

Good

Good

Good

Good

No UCT

Ins. data

Good

No UCT

Ins. data

No UCT 


$\begin{array}{lccl}\text { Apodemus agrarius } & 24.4 & 27.5 & \text { Liu } \text { et al., 2004 } \\ \text { Apodemus speciosus } & 28.5 & 30 & \text { Liu } \text { et al., 2004 } \\ \text { Thallomys paedulcus } & 124.7 & 35.89 & \text { Lovegrove } \text { et al., 1991 } \\ \text { Notomys cervinus } & 34.2 & 34 & \text { MacMillen \& Lee, 1970 } \\ \text { Notomys alexis } & 32.3 & 34 & \text { MacMillen \& Lee, 1970 } \\ \text { Pseudomys hermannsburgensis } & 12.2 & 36 & \text { MacMillen } \text { et al., 1972 } \\ \text { Cannomys badius } & 344 & 34.5 & \text { McNab, 1979 } \\ \text { Gerbillus pyramidum } & 53 & 31 & \text { Robinson, 1959 } \\ \text { Meriones unguiculatus } & 61 & 40 & \text { Robinson, 1959 } \\ \text { Aepyprymnus rufescens } & 2820 & 33 & \text { Rübsamen } \text { et al., 1984 } \\ \text { Acomys cahirinus } & 42 & 32.5 & \text { Shkolnik \& Borut, 1969 } \\ \text { Saccostomus campestris } & 61.3 & 32 & \text { Haim et al., 1991 } \\ \text { Steatomys pratensis } & 37.5 & 32 & \text { Perrin \& Richardson, 2005 } \\ \text { Octodon degus } & 206 & 35 & \text { Arends \& McNab, 2001 } \\ \text { Octodontomys gliroides } & 152 & 35 & \text { Arends \& McNab, 2001 } \\ \text { Spalacopus cyanus } & 185 & 34 & \text { Contreras, 1986; McNab, 1979 } \\ \text { Pedetes capensis } & 2300 & 33 & \text { Müller } \text { et al., 1979 } \\ \text { Spermophilus beecheyi } & 599.6 & 30 & \text { Baudinette, 1972 } \\ \text { Ammospermophilus leucurus } & 96 & 34 & \text { Dawson, 1955 } \\ \text { Tamiasciurus hudsonicus } & 231 & 27 & \text { Pauls, 1981 } \\ \text { Cynomys ludovicianus } & 1112.3 & 35 & \text { Reinking } \text { et al., 1977 } \\ \text { Spermophilus lateralis } & 257 & 30 & \text { Tattersall \& Milsom, 2003 } \\ \text { Tamias striatus } & 92.2 & 32 & \text { Wang \& Hudson, 1971 } \\ \text { Tamias minimus } & 50.6 & 34 & \text { Willems \& Armitage, 1975 } \\ \text { Tamias merriami } & 75 & 35 & \text { Wunder, 1970 } \\ & & & \\ & & & \end{array}$

\author{
Good \\ Good \\ Good \\ Good \\ Good \\ Ins. data \\ Good \\ No UCT \\ No UCT \\ No UCT \\ Ins. data \\ Ins. data \\ Ins. data \\ Ins. data \\ Ins. data \\ Good \\ Good \\ No UCT \\ Good \\ No UCT \\ Good \\ Good \\ Ins. data
}




\begin{tabular}{lcclc} 
Tachyoryctes splendens & 191 & 35 & McNab, 1966 & No UCT \\
Spalax leucodon & 207.7 & 34 & McNab, 1966 & No UCT \\
Tupaia belangeri & 186 & 35 & Zhang et al., 2012 & Good \\
Tupaia glis & 123 & 37 & Bradley \& Hudson, 1974 & No UCT \\
Blarina brevicauda & 20.5 & 33 & Deavers \& Hudson, 1981; Neal \& Lustik, 1973 & Ins. data \\
Neomys anomalus & 13.4 & 30 & Gebczynska \& Gebczynski, 1965 & Ins. data \\
Neomys fodiens & 14.2 & 30 & Gebczynska \& Gebczynski, 1965 & Ins. data \\
Notiosorex crawfordi & 4 & 36 & Lindstedt, 1980 & No UCT \\
Sorex minutus & 4.45 & 30 & McDevitt \& Andrews, 1995 & No UCT \\
Sorex cinereus & 3.5 & 30 & Morrison et al., 1959 & Ins. data \\
Neurotrichus gibbsii & 11.8 & 32 & Campbell \& Hochachka, 2000 & Ins. data \\
Condylura cristata & 49 & 33 & Campbell et al., 1999 & No UCT \\
Scalopus aquaticus & 48 & 33 & McNab, 1979 & Ins. data \\
\hline
\end{tabular}

